\title{
Technè
}

La science au service de l'histoire de l'art et de la préservation des biens culturels

$40 \mid 2014$

Thérapéia. Polychromie et restauration de la sculpture dans l'Antiquité

\section{Des dieux parmi les hommes : l'installation des «statues de culte » en Grèce ancienne}

Gods among men : the installation of "cult statues" in Ancient Greece

\section{Vinciane Pirenne-Delforge}

\section{OpenEdition}

10 Journals

Édition électronique

URL : https://journals.openedition.org/techne/3228

DOI : $10.4000 /$ techne.3228

ISSN : 2534-5168

Éditeur

C2RMF

Édition imprimée

Date de publication : 26 novembre 2014

Pagination : $30-34$

ISBN : 978-2-7118-6218-4

ISSN : 1254-7867

Référence électronique

Vinciane Pirenne-Delforge, «Des dieux parmi les hommes : I'installation des «statues de culte » en Grèce ancienne », Technè [En ligne], 40 | 2014, mis en ligne le 24 juillet 2020, consulté le 08 février 2022. URL : http://journals.openedition.org/techne/3228; DOI : https://doi.org/10.4000/techne.3228

\section{(c)}

La revue Technè. La science au service de l'histoire de l'art et de la préservation des biens culturels est mise à disposition selon les termes de la Licence Creative Commons Attribution - Pas d'Utilisation Commerciale - Pas de Modification 4.0 International. 


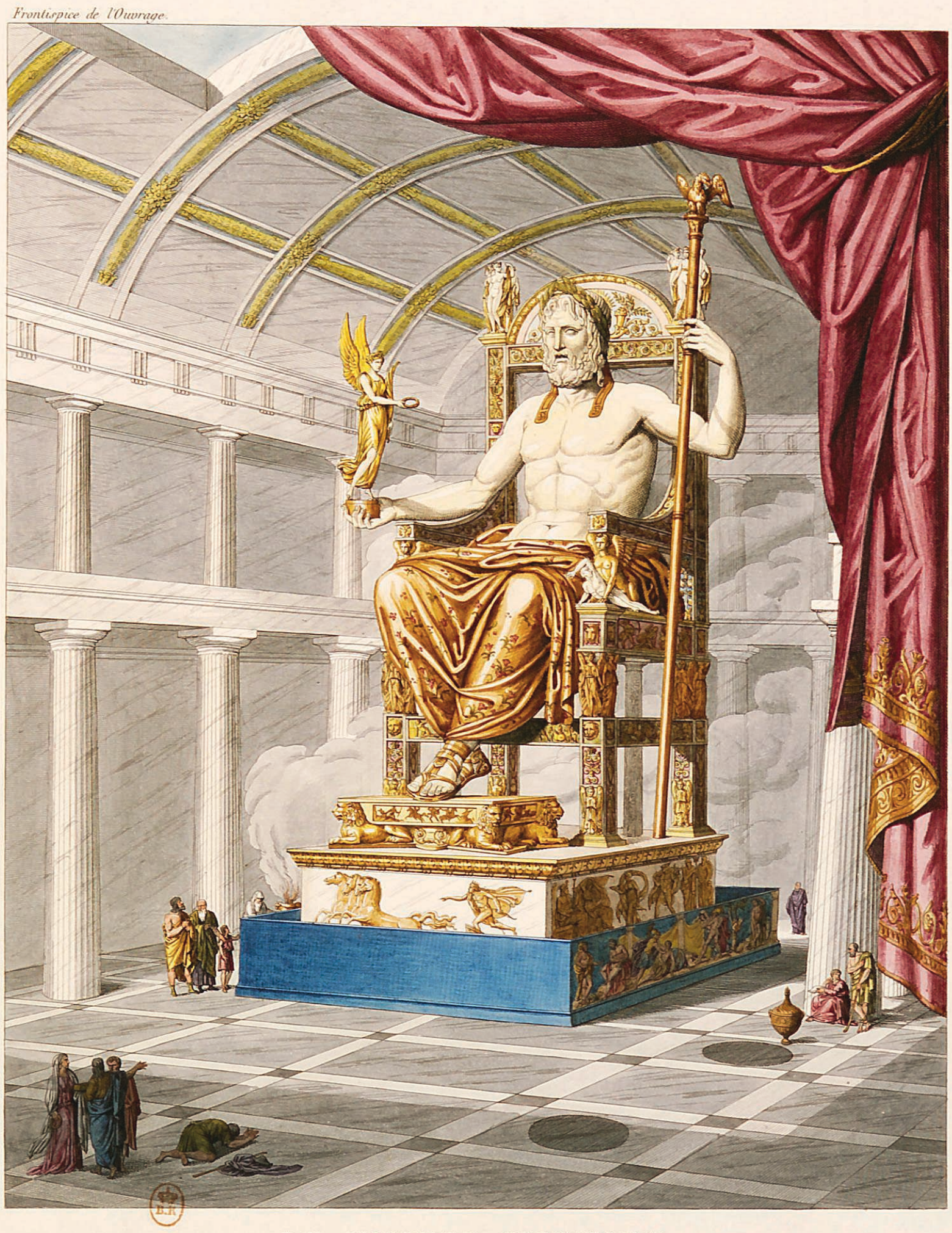

LE JUPITER OLYYIPIEN, 


\title{
Vinciane Pirenne-Delforge Des dieux parmi les hommes : l'installation des «statues de culte » en Grèce ancienne
}

\author{
in Ancient Greece
}

Résumé. La pluralité des dieux grecs et l'anthropomorphisme qui en caractérise la représentation ont donné lieu à la production de milliers de statues, dont une partie peuple aujourd'hui nos musées. Mais quel était le statut de ces statues dans le contexte qui les a vu naître? Qu'en est-il, parmi elles, des prétendues « statues de culte " ? La notion doit être interrogée en la replaçant dans le cadre de la représentation que les Grecs se faisaient de leurs dieux et de la manière dont ils entraient en communication avec eux par les rituels.

Mots-clés. Religion grecque, polythéisme, statues, Épiphanie, Phidias, Praxitèle, statue chryséléphantine, météorite.
Abstract. The plurality of Greek deities and the anthropomorphism that characterizes their representation gave rise to the production of thousands of statues, some of which are now housed in our museums. But what was the status of these statues in the context in which they emerged? What position did the so-called "cult statues" hold amongst them? This notion should be explored by placing it within the framework of how the Greeks depicted their divinities and how they communicated with them through rituals.

Keywords. Greek religion, polytheism, statues, Epiphany, Phidias, Praxiteles, chryselephantine statue, meteorite.
Le Zeus d'or et d'ivoire réalisé par Phidias pour le sanctuaire d'Olympie fait partie des statues antiques qui ont été vantées par les visiteurs de l'époque et ont stimulé l'imagination des modernes (fig. 1). Les anecdotes se sont multipliées à son propos dès l'Antiquité. Ainsi, à la question de savoir quel modèle allait servir pour représenter le dieu, Phidias aurait répondu que ce seraient les vers d'Homère évoquant la majesté du roi des dieux assis sur son trône et faisant trembler l'Olympe d'un froncement de sourcil ${ }^{1}$. Ensuite, au moment d'achever son œuvre, le sculpteur aurait prié le dieu de lui signifier si le travail était à son goût: la foudre serait alors venue frapper le sol au pied du colosse chryséléphantin. La trace de cette approbation divine était désormais marquée d'une hydrie de bronze $^{2}$. C'est au même Phidias qu'est attribuée l'affirmation des limites de la représentation d'une divinité, par nature incomparable et invisible, à laquelle l'artisan donne les formes visibles et comparables des statues, recourant dès lors à la force du symbole ${ }^{3}$.

Dans notre imaginaire moderne, le Zeus de Phidias devient ainsi une «statue de culte», à savoir la représentation du dieu à qui l'on rend hommage dans le sanctuaire où elle est installée. Mais cette catégorie ne va pas de soi en grec. Il n'existe pas de mot que l'on puisse traduire par «statue de culte». Le vocabulaire de la statuaire est pourtant varié dans la langue grecque ${ }^{4}$. On peut mentionner le terme de bretas, un mot ancien signifiant statue en bois, celui de xoanon, qui fait également référence au travail du bois, celui d'eikôn, orientant davantage vers la ressemblance, et donc vers le portrait, ou encore celui d'andrias, qui évoque une référence humaine. C'est néanmoins le terme d'agalma qui est le plus souvent utilisé dans les textes pour évoquer une statue divine en ronde-bosse, de taille plus ou moins humaine. Le sens premier du mot est celui de «bijou», d'«ornement», de «cadeau». L'agalma est avant tout un présent fait à une divinité, pour lui plaire, l'honorer, la solliciter, la remercier. Par extension, le terme s'est appliqué de façon privilégiée à toute statue divine, conçue comme «le cadeau » par excellence. Il est dès lors erroné de toujours traduire agalma par «statue de culte»: il s'agit avant tout d'une statue divine.

Contrairement à d'autres cultures, notamment procheorientales, la Grèce ne connaît pas de rituel de consécration spécifique qui fonde le statut particulièrement divin de la représentation d'un dieu dans le sanctuaire qui lui est dévolu: pas d'ouverture symbolique de la bouche de la statue, pas de manipulation visant à incorporer le dieu dans sa statue ${ }^{5}$. Toutefois, bien des témoignages sur les statues divines invitent à considérer les diverses manières dont se traduit le rapport au dieu qu'elles sont censées évoquer.

La dédicace dans un sanctuaire est le processus le plus commun, ce qui fait d'une statue la «propriété du dieu». L'agalma entre alors dans la catégorie très vaste des anathèmata, offrandes dédicatoires déposées dans un lieu sacré afin d'honorer la divinité, attirer sa bienveillance ou la remercier d'un bienfait. Une telle «déposition» peut être conçue comme une forme de consécration, dans le sens où la propriété divine est sacrée.

Par ailleurs, il est des cas où une statue, parmi celles qui s'élèvent dans un sanctuaire, voire dans un temple, est chargée 
d'une dimension sacrée particulière: les récits qui la concernent et les manipulations dont elle fait l'objet dessinent l'aura spécifique qui l'entoure. C'est par exemple le cas de l'antique statue en bois d'Athéna Polias sur l'acropole d'Athènes. Les Athéniens considéraient qu'elle était tombée du ciel ${ }^{6}$, et donc envoyée par la déesse elle-même. Elle fut précieusement conservée, en dépit du saccage de la cité par les Perses en 480 avant notre ère ${ }^{7}$ : c'est à cet objet vénérable qu'était destiné le fastueux péplos brodé par des femmes et des jeunes filles d'Athènes, et offert à la déesse tutélaire de la cité tous les quatre ans lors des Grandes Panathénées. Les récits et les rituels qui entourent l'Artémis de Pellène dans le nord du Péloponnèse sont tout aussi édifiants. Seule la prêtresse était habilitée à manipuler la statue. Quand elle transportait l'objet hors du temple, personne ne pouvait la regarder, car la représentation de la déesse était dangereuse tant pour les humains que pour les végétaux. Lors d'une bataille contre les Étoliens, la prêtresse aurait menacé les ennemis en sortant la statue du temple et les aurait ainsi rendus fous ${ }^{8}$.

On pourrait multiplier les exemples de traditions suscitées par ces objets remarquables, considérés comme les réceptacles d'une puissance divine particulièrement active, liée à la dimension épiphanique qui entoure leur origine ou leur action en contexte rituel. Ainsi les statues en bois qui font l'objet de manipulations (habillage et déshabillage, transport en procession, lavage dans un fleuve ou dans la mer, disparition puis retour, etc.). Si la mise en scène de ces objets était parfois liée à des impératifs concrets d'entretien, il ne faudrait pas appliquer à cette démarche une interprétation strictement matérielle. L'ostentation de la statue ou, au contraire, le secret qui entourait les soins qu'elle recevait faisaient partie des temps forts du calendrier des fêtes d'une communauté et contribuait à réaffirmer sa communication avec le divin ${ }^{9}$. Au II ${ }^{\mathrm{e}}$ siècle de notre ère, le voyageur érudit Pausanias a conservé le souvenir de ce type de tradition, en associant aux antiques statues en bois une intensité divinement inspirée qui compensait largement le caractère frustre de leur apparence ${ }^{10}$.

Dans le cas des anathèmata, c'est le contexte où la déposition s'effectue qui sacralise l'objet et le soustrait à tout autre usage. Déplacer des offrandes hors d'un sanctuaire ou les transformer sans précaution relève du sacrilège. Les statues de type épiphanique sont également des propriétés divines, mais la tradition qui les accompagne leur confère un surcroît de dignité - comme un «supplément d'âme»-, et donc une attention différente de celle accordée aux simples offrandes dédicatoires, fussent-elles l'œuvre de sculpteurs célèbres ${ }^{11}$. C'est la raison pour laquelle certains types d'objets non anthropomorphes, comme des fragments de météorite, peuvent recevoir un culte lié à une divinité: par exemple, à Orchomène de Béotie, le culte des trois Charites se concentrait autour de pierres qui étaient dites tombées du ciel aux premiers temps de la cité ${ }^{12}$.

En faisant tomber du ciel sa propre statue, une divinité est censée créer elle-même la communication autour de l'objet. Les météorites et autres objets extraordinaires ont une telle vertu. Ils sont de nature épiphanique. Cependant, toutes les communautés, tous les sanctuaires n'avaient pas la chance de posséder une statue ou un objet de ce type. Il leur fallait dès lors passer commande auprès d'un sculpteur, plus ou moins réputé selon les cas. On a vu que la cité d'Élis, en charge du sanctuaire d'Olympie, s'était offert les services de Phidias. Par la suite, ses descendants - ou supposés tels - ont eu la charge d'entretenir la statue, assumant la fonction de «lustreurs» (phaidryntai) ${ }^{13}$. La bonne tenue de l'ivoire requérait des dispositifs spécifiques ${ }^{14}$, mais l'option chryséléphantine était exceptionnelle. Le bronze et le marbre étaient davantage utilisés, mais on n'a pas conservé un quelconque «bon de commande» ni l'«appel d'offre» que les cités devaient sans doute soumettre aux sculpteurs. Seule une anecdote permet de saisir une toute petite part du processus. Pline l'Ancien raconte que la cité de Cos s'était adressée à Praxitèle pour concevoir la statue d'Aphrodite d'un sanctuaire local. Le sculpteur aurait proposé deux figurations en marbre aux commanditaires : l'une voilée et l'autre nue. Les gens de Cos choisirent la version voilée, plus austère et plus chaste. Les gens de Cnide auraient alors acquis l'Aphrodite nue, qui devait faire ensuite la réputation de leur cité ${ }^{15}$. Cependant, avant d'être un objet de curiosité, voire de désir, l'Aphrodite nue de Praxitèle était un objet de culte. Comment ce statut lui était-il conféré?

La même question se pose lorsqu'on trouve juxtaposés, dans un sanctuaire, une antique statue en bois (un xoanon) et un chef-d'œuvre "contemporain", figurant tous deux une même divinité ${ }^{16}$. Comment comprendre le statut respectif de ces objets? Dans le sanctuaire d'Artémis à Brauron, l'un des dèmes de l'Attique en bord de mer, tant les inscriptions que les découvertes archéologiques permettent de reconstituer quatre statues acrolithes de la déesse. Les inventaires mis au jour dans le sanctuaire que l'Artémis Brauronia possédait également sur l'acropole d'Athènes témoignent que des vêtements étaient destinés à certaines de ces statues, dont seules les extrémités étaient en marbre ${ }^{17}$. Mais quel était le statut exact de ces acrolithes? Qu'il s'agisse d'anathèmata est une certitude. Peut-on aller plus loin et parler de «statues de culte»? De même, quand un xoanon et une statue «moderne» cohabitent dans un temple, a-t-on affaire à deux «statues de culte»?

On a vu que la manifestation divine était au cœur de la définition d'un objet épiphanique: l'action posée par le dieu lui confère ce «quelque chose de divin » qui transcende même un morceau de bois mal dégrossi ou une pierre brute. C'est donc la manifestation divine qui ouvre la voie à une communication avec la communauté par la médiation d'un objet. Il convient d'explorer cette voie d'interprétation en élargissant la perspective adoptée jusqu'ici.

En effet, les dieux ne sont pleinement divins que si les hommes leur rendent hommage. Ce fondement de la représentation grecque du divin se traduit dans bon nombre de récits mettant en évidence l'intérêt que les dieux prêtent aux cités des hommes et leur souci de recevoir la somme des honneurs qui leur sont dus. C'est sur cet arrière-plan que doivent se comprendre les querelles qui auraient opposé 
certains dieux en vue de la possession de telle ou telle cité. Le cas le plus connu est celui de la dispute entre Athéna et Poséidon à Athènes ${ }^{18}$, figurée au fronton du Parthénon. Mais ce n'est pas le seul ${ }^{19}$. Dans sa critique acerbe d'une telle représentation des dieux, Lucien décrit adéquatement le processus du point de vue humain : les hommes se répartissent les dieux, les honorent et les revendiquent comme leurs concitoyens ${ }^{20}$. Il s'agit d'une caricature, mais l'image est ancrée dans l'imaginaire des Grecs et le vocabulaire en témoigne. Car s'il n'existe pas de procédure clairement identifiée, à l'échelle du polythéisme grec, pour «consacrer une statue», un champ sémantique se dégage pour dire cette cohabitation entre les hommes et les dieux: c'est celui de "l'installation".

Dès la période classique, fonder un culte se dit au moyen de termes de la famille d'hidruein, «installer, fonder, établir». La procédure vaut tant pour les temples et les autels que pour les statues. Ce sont ces dernières qui nous intéressent ici. Ainsi, dans l'Iphigénie en Tauride, Euripide met en scène Oreste poussé par Apollon à rechercher l'antique image d'Artémis. L'oracle du dieu a stipulé au héros qu'il devrait ériger (enkathidruein) la statue en terre athénienne ${ }^{21}$. De même, sur un mode plus léger, Aristophane utilise ce registre sémantique dans deux de ses comédies. Dans La Paix, c'est cette dernière, divinisée, qui avait été enlevée par Polémos, «la Guerre», et qu'il faut «réinstaller ${ }^{22}$ ». Dans le Ploutos, c'est ce dernier, divinisation de la Richesse, qu'il convient de réinstaller dans l'opisthodome du temple d'Athéna qu'il n'aurait jamais dû quitter $^{23}$. À chaque fois, les indications du texte laissent entendre que les acteurs se réfèrent à une statue. À chaque fois, l'expression utilisée est concrète: la divinité sera «installée par des marmites» (chutrais hidruein). Les scholies aux textes explicitent ce qui devait être évident pour les spectateurs de ces comédies : on pouvait installer un dieu en faisant bouillir des céréales dans des marmites ou en choisissant une offrande plus coûteuse. D'où l'expression complémentaire : «Installer par un bœuf, par une chèvre ou par du menu bétail ${ }^{24}$.»

L'«installation par des marmites » est une pratique dont ces gloses attestent le caractère modeste et rapide, d'une part, mais aussi l'équivalence structurelle avec une immolation animale. L'opération s'inscrit ainsi dans le registre sacrificiel. Or, sacrifier à un dieu est, par excellence, la manière d'entrer en contact avec lui, tout en l'honorant comme il se doit. Dès lors, la communication est un objectif fondamental dans la procédure d'installation du dieu parmi les hommes. L'objectif du rituel est d'intégrer le dieu à la cité ou au groupe, de créer les conditions de la manifestation de sa divinité, de sa bienveillance au cœur même de l'espace de vie de ceux qui l'honorent. La statue - comme l'autel, lui aussi «installé »-devient alors le lieu privilégié de cette manifestation du divin et le sacrifice en est l'instrument. La procédure d'installation ouvre la voie de la communication en créant les conditions privilégiées de l'interaction à venir entre hommes et dieux. Le rituel active la bienveillance divine et la statue devient un «objet de culte». Toutefois, aucun terme grec n'est habilité à saisir une fois pour toutes l'objet ainsi fondé, installé. D'où l'impossibilité de retrouver la «statue de culte» dans le lexique grec ${ }^{25}$.

Une statue de type épiphanique et une statue installée sont toutes deux des lieux privilégiés de la manifestation du divin pour la communauté qui est dépositaire des traditions liées à la première et pour celle qui est à l'initiative du processus de fondation de la seconde. Une des anecdotes sur le Zeus de Phidias, évoquée en ouverture, est significative à cet égard. En effet, le colosse chryséléphantin n'est pas, en soi, une statue «épiphanique». C'est une ouvre d'art de la période classique, c'est-à-dire une œuvre «moderne», même pour Pausanias qui l'admire près de sept siècles après sa mise en place. Mais l'éclair qui s'abat au pied de la statue en réponse à la recherche d'approbation de Phidias est de l'ordre de l'épiphanie divine. Et - qui sait? - peut-être l'hydrie qui marquait le sol où le dieu s'était prétendument manifesté avait-elle fait partie du rituel «d'installation» de la statue dans son sanctuaire, à Olympie, à la fin du $\mathrm{V}^{\mathrm{e}}$ siècle avant notre ère. Rituel d'installation et tradition épiphanique en seraient venus à se mêler ensuite, jusqu'à ce qu'un visiteur curieux en recueille le témoignage sous les Antonins.

\section{Notes}

Liste des sigles et abréviations de collections

ID: Corpus des Inscriptions de Délos, Paris. $I G$ : Inscriptiones Graecae, Berlin.

Cet article est adapté d'une étude antérieure sur le sujet : Pirenne-Delforge, 2008.

1. Strabon, VIII, 30, 3.

2. Pausanias, V, 11, 9.

3. Dion Chrysostome, Discours, 12, 59. Voir Collard, 2013.

4. Scheer, 2000, p. 3-34; Bettinetti, 2001, p. 25-63. Voir Donohue, 1997, pour une perspective historiographique qui aboutit à reléguer la notion de "statue de culte» au magasin des idées reçues non pertinentes.
5. Rudhardt, 2001, p. 175-188; Graf, 2001, p. 230

6. Pausanias, I, 26, 6.

7. Plutarque, Thémistocle, $10,7$.

8. Plutarque, Aratos, 32, 2.

9. Bettinetti, 2001, p. 137-210.

10. Pausanias, II, 4, 5 .

11. Pausanias, I, 43, 6 .

12. Pausanias, IX, 38, 1 .

13. Pausanias, V, 14, 5.

14. Pausanias, V, 11, 10 : à Olympie, on verse de l'huile autour de la statue de Zeus; à Athènes, c'est de l'eau qui permet d'humidifier l'ivoire de l'Athéna Parthénos sur une acropole particulièrement aride; à Épidaure, la statue chryséléphantine d'Asclépios assis sur un trône a été établie sur un puits. Sur ces statues: Lapatin, 2001.

15. Pline, Histoire naturelle, XXXVI, 20.
16. Par exemple, IG XII 6, 261 (Samos, $346 / 5$ av. n. è.), où il est fait référence à deux statues d'Héra: lignes 19, 28, 29, 32 (ỉ Өєós), 27 (†่ óm $1 \sigma \theta \varepsilon \theta \varepsilon o ́ \varsigma)$. On sait par ailleurs que l'une était en bois.

17. Despinis, 2004.

18. Euripide, Phéniciennes, 854;

Hérodote, VIII, 55; Pausanias, I, 26, 5.

19. Darthou, 2008.

20. Lucien, Sacrifices, 10.

21. Euripide, Iphigénie à Aulis, 976-978.

22. Aristophane, Paix, 926-938.

23. Aristophane, Ploutos, 1191-1199.

24. Souda, s.v. "Xútpaıs ífputéov» (éd.

Adler IV [1935], p. 836).

25. Scheer, 2000, passim. 


\section{Bibliographie citée}

Bettinetti S., 2001, La statua di culto nella pratica rituale greca, Bari.

Collard H., 2013, «Montrer l'invisible. Les dieux et leurs statues dans la céramique grecque ", dans Borgeaud Ph., Fabiano D. (éd.), Perception et construction du divin dans l'Antiquité, Genève, p. 61-86.

Darthou S., 2008, «Éris dans la cité. Quelques réflexions sur les

"cosmogonies" politiques ", Mètis, N.S., p. 269-285.

Despinis G. I., 2004, «Die Kultstatuen der Artemis in Brauron ", $M D A I(A)$ 119, p. 261-315.
Donohue A. A., 1997, “The Greek Images of the Gods. Considerations on terminology and methodology", Hephaistos 15, p. 31-45.

Graf F., 2001, «Der Eigensinn der Götterbilder in antiken religiösen Diskursen », dans G. Boehm (éd.), Homo Pictor, Leipzig (Colloquium Rauricum, Band 7), p. 227-243.

Lapatin K., 2001, Chryselephantine Statuary in the Ancient Mediterranean World, Oxford

Pirenne-Delforge V., 2008, «Des marmites pour un méchant petit hermès, ou comment consacrer une statue ", dans Estienne S., Jaillard D., Lubtschansky N., Pouzadoux C. (éd.), Image et religion dans l'Antiquité gréco-romaine. Actes du Colloque de Rome, 11-13 décembre 2003, Naples, p. 103-110.

Rudhardt J., 2001, «La perception grecque du territoire sacré », MEFRA 113, p. 175-188.

Scheer T., 2000, Die Gottheit und ihr Bild. Untersuchungen zur Funktion griechischer Kultbilder in Religion und Politik, Munich. 